\title{
COMMENTARY
}

\section{Breaking the Barriers}

\section{New Role for Insulin-Like Growth Factor 1 Receptor in Vascular Permeability}

\author{
Sandhya Xavier
}

From the Department of Medicine, Renal Research Institute, New York Medical College, Valhalla, New York

The potential contribution of endothelial barrier function in chronic kidney disease and renal fibrosis remains largely unexplored and represents an exciting problem to be studied in nephrology research.

The vascular endothelium forms a selective barrier between the blood stream and the underlying tissues; its permeability is mediated by transcellular and paracellular pathways. Coordinated opening and closure of endothelial cell (EC)-cell junctions regulates paracellular permeability. ${ }^{1,2}$

EC junction stability in established vessels is maintained by several adhesion molecules and receptors that are organized into tight junctions and adheren junctions. Adheren junctions are formed by members of the cadherin family of adhesion proteins. ECs are known to express high amounts of the cadherins: VE (vascular endothelial) expressed only in ECs and $\mathrm{N}$-cadherins (neuronal) that are also present in other cell types. ${ }^{3}$ The importance of VE-cadherin in the maintenance of vascular integrity is demonstrated by the fact that administration of anti-VE-cadherin antibodies leads to an increase in permeability, vascular fragility, and hemorrhages in the adult mouse. ${ }^{4}$

Endothelial barrier function can be reinforced by strengthening connections between adheren junctions and actin cytoskeleton. These studies were conducted by Vestweber and colleagues $^{5}$ whereby they demonstrated that mice in which VE-cadherin is replaced with a VE-cadherin- $\alpha$-catenin fusion construct exhibit resistance to vascular leaks by VE growth factor and histamine, and the recruitment of neutrophils and leukocytes at inflammatory sites is strongly impaired.

Another mechanism that modulates vascular permeability is tyrosine phosphorylation of VE-cadherin. VE-protein tyrosine phosphatase (PTP), an endothelial-specific phosphatase, associates with VE-cadherin to inhibit tyrosine phosphorylation. ${ }^{6}$
Its dissociation opens EC contacts during induction of vascular permeability and leukocyte extravasation. ${ }^{7}$ Subsequently, in a knock-in mouse that carries a tyrosine-tophenylalanine point mutation of VE-cadherin, it was found that phosphorylation of this tyrosine residue is a major regulator of endothelial integrity in vivo, especially in organs such as the ovary and uterus because they developed edema and fibrosis both of which are hallmarks of increased vascular permeability. ${ }^{8}$

The dissociation of VE-PTP from VE-cadherin can be induced by VE growth factor, lipopolysaccharide-induced leukocyte extravasation, and lymphocytes. ${ }^{9}$ It is not known if other potential factors can trigger this dissociation. In the current issue of The American Journal of Pathology, Liang et $\mathrm{al}^{10}$ describe a novel role for insulin-like growth factor 1 receptor (IGF-1R) in maintaining vascular permeability and how its expression affects endothelial function and the progression of chronic kidney disease. The rationale for this study is based on the compelling evidence that IGF-1R is a vascular protective factor and is abundantly expressed in ECs. ${ }^{11,12}$ The authors clearly demonstrate that loss of IGF$1 \mathrm{R}$ in vascular ECs increases permeability, infiltration of inflammatory cells, and progressive renal interstitial fibrosis in the unilateral ureteral obstruction model of chronic kidney disease.

The authors evaluated dysfunction of the endothelial barrier in mice with selective deletion of IGF-1R in the endothelium after unilateral ureteral obstruction by measuring

Accepted for publication February 12, 2015.

Disclosures: None declared.

Address correspondence to Sandhya Xavier, Ph.D., Department of Medicine, Renal Research Institute, New York Medical College, 15 Dana Rd., Valhalla, NY 10595. E-mail: sandhya_xavier@nymc.edu. 
deposition of Evans Blue dye in the renal interstitium and further showed by transmission electron microscopy severe loss of cellular junctions in knockout mice associated with a significant increase in VE-cadherin protein phosphorylation. The presence of a leaky EC barrier was accompanied by increased platelet aggregation in the interstitial capillary (CD-42 staining) of the obstructed kidneys and increased inflammatory cell leakage. Collectively, these novel findings suggest that IGF-1R in ECs plays a critical role in maintaining vascular integrity by stabilization of VE-cadherin at endothelial junctions. Moreover, restoration of IGF-1R expression in ECs not only ameliorates inflammatory cell infiltration but also suppresses renal fibrosis.

IGF-1R belongs to the receptor tyrosine kinase family and comprises a $\alpha_{2} \beta_{2}$ heterotetrameric complex, with two extracellular subunits coupled to the membrane-spanning $\beta$ subunit, which contains an intracellular domain with intrinsic tyrosine kinase activity. ${ }^{13}$ Binding of IGF-1 to its receptor initiates a complex of canonical and noncanonical signaling cascades. One main role of IGF-1R in ECs appears to be the modulation of insulin-stimulated nitric oxide production via activation of phosphatidylinositol 3-kinaseAkt-endothelial nitric oxide synthase pathway. ${ }^{14}$ Hybrid receptors composed of insulin receptors (IRs) and IGF-1Rs were identified in human ECs. ${ }^{15}$ Rodent models of obesity and diabetes exhibit increased expression of IGF-1R. ${ }^{12}$ Higher IGF-1R expression is associated with increased formation of IR/IGF-1R and a lower proportion of IR holoreceptors that ultimately lead to a lower magnitude of nitric oxide production. ${ }^{16}$ Importantly, the insulin and IGF signaling system has become a much sought after therapeutic target for diabetes and cancer because these receptors are overexpressed in a variety of cancers, including prostate and breast cancers. ${ }^{17,18}$ Although its increase is associated with diabetes and cancer, the loss of IGF-1R in ECs increases vascular permeability associated with renal fibrosis as shown by Liang et $\mathrm{al}^{10}$ in this issue and is also linked to diabetes-induced cardiac fibrosis. ${ }^{19,20}$ These opposing effects due to altered receptor expression amount bring up the question, what distinguishes the outcome? It appears that a tight and physiologically relevant critical range of IGF-1R expression is essential to relay its biological effects. Anything above or below this range resets the balance. Moreover, although an increase in IGF-1R expression increases the risk of diabetes, it has a protective effect in enhancing endothelial repair and regeneration. ${ }^{16}$

The results from the study by Liang et $\mathrm{al}^{10}$ demonstrate for the first time the involvement of IGF-1R in stabilizing VEcadherin/VE-PTP complex at adheren junctions. The question that needs clarity is whether insulin receptors are also a player in this complex, because the IGF-1R/IR hybrid receptor stoichiometry has significant biological relevance particularly in ECs, thus making it a complex system with multiple interacting partners. In addition, what happens to VE-cadherin/ VE-PTP complex and phosphorylation of VE-cadherin during ligand-mediated receptor activation remains unanswered.
Additional studies are particularly needed to understand the mechanism by which IGF-1R loss occurs during renal injury. The authors indeed elegantly demonstrate that its ablation in ECs is sufficient to promote renal fibrosis, exaggerated deposition of interstitial collagen accompanied by infiltration of inflammatory cells in interstitial areas surrounding vessels. Further, when they overexpressed IGF$1 \mathrm{R}$ in ECs, a significant reduction in inflammatory cell infiltration in the obstructed kidneys of IGF-1R transgenics with reduced fibrosis and better endothelial barrier function was observed. In vitro experiments that use primary cell cultures also support their in vivo hypothesis and clearly demonstrate the involvement of IGF-1R in stabilizing the VE-cadherin complex. Interestingly, it was recently shown in a VE-cadherin knock-in mouse, which is resistant to phosphorylation at tyrosine 685 , that there are spontaneously leaky capillaries compared with wild-type mice. ${ }^{8}$ These observations lead to speculation of the effect of phosphorylation on endothelial barrier function. Whether the endothelial dysfunction as observed in the IGF-IR mutant mice after renal injury is a direct effect of phosphorylation of VEcadherin or its dissociation from VE-PTP still remains unclear and warrants further investigations. Despite these limitations the findings of this study are novel. The current work suggests that IGF-1R amounts play a critical role in maintaining endothelial barrier function, and its relevance in clinical samples needs to be explored further so that strategies to improve endothelial barrier function to reduce progression of chronic kidney disease can become future directions.

\section{References}

1. Dejana E: Endothelial cell-cell junctions: happy together. Nat Rev Mol Cell Biol 2004, 5:261-270

2. Vestweber D: Adhesion and signaling molecules controlling the transmigration of leukocytes through endothelium. Immunol Rev 2007, 218:178-196

3. Bazzoni G, Dejana E: Endothelial cell-to-cell junctions: molecular organization and role in vascular homeostasis. Physiol Rev 2004, 84: 869-901

4. Corada M, Mariotti M, Thurston G, Smith K, Kunkel R, Brockhaus M, Lampugnani MG, Martin-Padura I, Stoppacciaro A, Ruco L, McDonald DM, Ward PA, Dejana E: Vascular endothelial-cadherin is an important determinant of microvascular integrity in vivo. Proc Natl Acad Sci U S A 1999, 96:9815-9820

5. Schulte D, Küppers V, Dartsch N, Broermann A, Li H, Zarbock A, Kamenyeva O, Kiefer F, Khandoga A, Massberg S, Vestweber D: Stabilizing the VE-cadherin-catenin complex blocks leukocyte extravasation and vascular permeability. EMBO J 2011, 30:4157-4170

6. Nawroth R, Poeli G, Rauft A, Kloep S, Samulowitz U, Fachinger G, Golding M, Shima DT, Deutsch U, Vestweber D: VE-PTP and VEcadherin ectodomains interact to facilitate regulation of phosphorylation and cell contacts. EMBO J 2002, 21:4885-4895

7. Broermann A, Winderlich M, Block H, Frye M, Rossaint J, Zarbock A, Cagna G, Linnepe R, Schulte D, Nottebaum AF, Vestweber D: Dissociation of VE-PTP from VE-cadherin is required for leukocyte extravasation and for VEGF-induced vascular permeability in vivo. J Exp Med 2011, 208:2393-2401

8. Sidibé A, Polena H, Pernet-Gallay K, Razanajatovo J, Mannic T, Chaumontel N, Bama S, Maréchal I, Huber P, Gulino-Debrac D, 
Bouillet L, Vilgrain I: VE-cadherin Y685F knock-in mouse is sensitive to vascular permeability in recurrent angiogenic organs. Am J Physiol Heart Circ Physiol 2014, 307:H455-H463

9. Vockel M, Vestweber D: How T cells trigger the dissociation of the endothelial receptor phosphatase VE-PTP from VE-cadherin. Blood 2013, 122:2512-2522

10. Liang M, Woodard LE, Liang A, Luo J, Wilson MH, Mitch WE, Cheng J: Protective role of IGF-1R in endothelial cells against UUO-induced renal fibrosis. Am J Pathol 2015, 185:1234-1250

11. Chisalita SI, Arnqvist HJ: Insulin-like growth factor I receptors are more abundant than insulin receptors in human micro- and macrovascular endothelial cells. Am J Physiol Endocrinol Metab 2004, 286: E896-E901

12. Delafontaine P, Song YH, Li Y: Expression, regulation, and function of IGF-1, IGF-1R, and IGF-1 binding proteins in blood vessels. Arterioscler Thromb Vasc Biol 2004, 24:435-444

13. Adams TE, Epa VC, Garrett TP, Ward CW: Structure and function of the type 1 insulin-like growth factor receptor. Cell Mol Life Sci 2000, 57:1050-1093

14. Zeng G, Quon MJ: Insulin-stimulated production of nitric oxide is inhibited by wortmannin. Direct measurement in vascular endothelial cells. J Clin Invest 1996, 98:894-898
15. Belfiore A, Frasca F, Pandini G, Sciacca L, Vigneri R: Insulin receptor isoforms and insulin receptor/insulin-like growth factor receptor hybrids in physiology and disease. Endocr Rev 2009, 30:586-623

16. Imrie H, Viswambharan H, Sukumar $\mathrm{P}$, Abbas A, Cubbon RM, Yuldasheva N, Gage M, Smith J, Galloway S, Skromna A, Rashid ST, Futers TS, Xuan S, Gatenby VK, Grant PJ, Channon KM, Beech DJ, Wheatcroft SB, Kearney MT: Novel role of the IGF-1 receptor in endothelial function and repair: studies in endothelium-targeted IGF-1 receptor transgenic mice. Diabetes 2012, 61:2359-2368

17. Gualberto A, Pollak M: Emerging role of insulin-like growth factor receptor inhibitors in oncology: early clinical trial results and future directions. Oncogene 2009, 28:3009-3021

18. Rodon J, DeSantos V, Ferry RJ Jr, Kurzrock R: Early drug development of inhibitors of the insulin-like growth factor-I receptor pathway: lessons from the first clinical trials. Mol Cancer Ther 2008, 7:2575-2588

19. Huynh K, McMullen JR, Julius TL, Tan JW, Love JE, Cemerlang N, Kiriazis H, Du XJ, Ritchie RH: Cardiac-specific IGF-1 receptor transgenic expression protects against cardiac fibrosis and diastolic dysfunction in a mouse model of diabetic cardiomyopathy. Diabetes 2010, 59:1512-1520

20. Troncoso R, Ibarra C, Vicencio JM, Jaimovich E, Lavandero S: New insights into IGF-1 signaling in the heart. Trends Endocrinol Metab 2014, 25:128-137 